\title{
Unearthing roles for the gut microbiome
}

\section{4 \\ significant differences in $\beta$-diversity were noted according to ADT status}

The gut microbiome is linked to a number of lifestyle factors that contribute to prostate cancer development and progression, including obesity and diet. Furthermore, it has known roles in metabolism of chemotherapeutics, contributing to drug resistance.

In a study published in European Urology, Liss and co-workers carried out microbiome analysis using $16 \mathrm{~S}$ ribosomal RNA (rRNA) amplicon sequencing on faecal samples from 105 men, 64 of whom were subsequently diagnosed with prostate cancer and 41 who were not. $16 \mathrm{~S}$ rRNA sequences were assigned a taxonomic lineage, and Phylogenetic Investigation of Communities by Reconstruction of Unobserved States (PICRUSt) analysis was used to identify metabolic pathways affected by groups of bacteria. No association between gut microbiome diversity and cancer status was observed; however, the team did find significant associations between total community composition and cancer status, showing that the majority of taxa were similar between patients with and without prostate cancer. Bifidobacteria usually associated

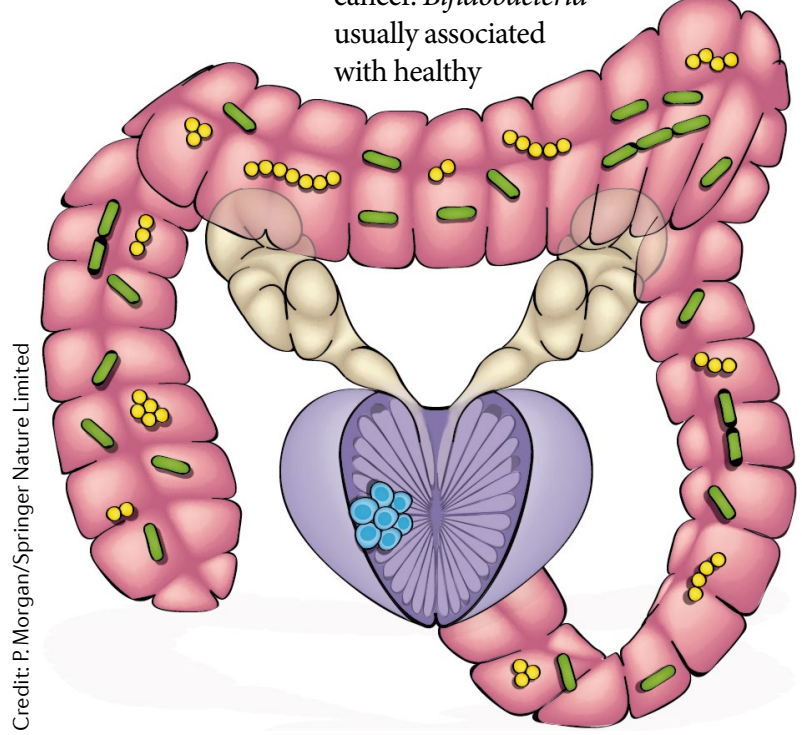

gut flora - were rare in both groups, but significant differences in Bacteroides and Streptococcus were observed between the groups and bacteria associated with carbohydrate metabolism were abundant. PICRUSt suggested a correlation between cancer status and B vitamins; folate and arginine pathways were the most significantly altered in men with prostate cancer. As the gut microbiome is modifiable, manipulations such as improving natural bacterial folate production might be possible to improve prostate cancer risk.

Finally, the team used a microbiome metabolic pathway score to predict future prostate cancer risk. This score was the only significant factor in univariate analysis that predicted future cancer, even controlling for age and PSA, despite a suboptimal AUC (0.64).

A second study, published in Prostate Cancer and Prostatic Diseases, investigated compositional differences in the gut microbiota of patients with prostate cancer who had undergone androgen-targeting therapy. Sfanos and colleagues profiled the faecal microbiome of 30 men including healthy controls and men with various stages of prostate cancer, including metastatic disease. In contrast to Liss et al., significant differences in $\alpha$-diversity were observed according to cancer status, which were likely due, in part, to the Liss cohort including only men with primary prostate cancer, whereas the Sfanos cohort included many men with advanced disease. Species richness and the presence of rare individual species were greater in those without a cancer diagnosis, regardless of whether they had received androgen deprivation therapy (ADT); significant differences in $\beta$-diversity were noted according to ADT status. Further investigation revealed that Akkermansia muciniphila, Ruminococcaceae spp., and Lachnospiraceae spp. were more abundant in samples from the oral ADT group than from men not receiving $A D T$, with a decrease in abundance of Brevibacteriaceae, Erysipelotrichaceae, and Streptococcaceae. These data are particularly interesting as A. muciniphila has been associated with positive responses to anti-programmed cell death 1 (PD-1) immunotherapy. The authors speculate that responses to anti-PD-1 therapy in men who have progressed on enzalutamide might be related to abundance of this species.

Finally, PICRUSt analysis showed that pathways involving steroid hormone biosynthesis were enriched in the oral ADT group compared with that of men not receiving ADT, suggesting that bacterial species capable of steroid biosynthesis might be more abundant in the gut flora of men taking oral ADT, potentially influencing treatment response.

These studies raise the question of whether the gut microbiome has a role in treatment efficacy and/ or whether it could be targeted to improve treatment responses in men with prostate cancer. "The immediate next question is 'do the changes we see correlate with treatment response?"' comments lead author Karen Sfanos. "To address this, we are currently scaling up the project to a much larger cohort as part of a study funded by the Prostate Cancer Foundation."

Annette Fenner

ORIGINAL ARTICLES Liss, M. A. et al. Metabolic biosynthesis pathways identified from fecal microbiome associated with prostate cancer Eur.Urol. https://doi.org/10.1016/j.eururo.2018. 06.033 (2018) | Sfanos, K. S. et al. Compositional differences in gastrointestinal microbiota in prostate cancer patients treated with androgen axis-targeted therapies. Prostate Cancer Prostatic Dis. https://doi.org/10.1038/s41391-018-0061-x (2018) 